\title{
Training of caregiver for home care management of stroke survivor at low resource setting
}

\author{
Md. Shahidur Rahman and Abul Khair Mohammad Salek
}

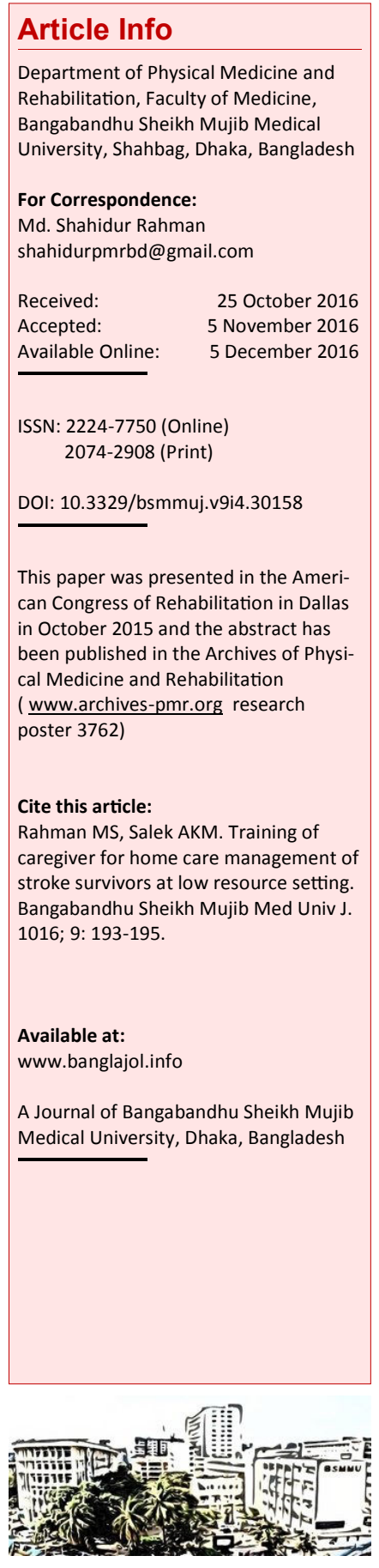

\begin{abstract}
This study was carried out to see whether caregiver training to attendees during hospital stay can better address the stroke survivor at home. In total 119 admitted patients hailed from remote villages were randomly selected from a private neurology hospital in Dhaka and were divided into two groups. One group had caregiver training and another group without training. All the patients were assessed for Barthel index at discharge and after 2 months. Barthel index in Group A during discharge was $6.2 \pm 4.0$ and at follow-up was $56.4 \pm 9.0$. The index in Group B was $4.9 \pm 4$ during discharge and $28.2 \pm 7.8$ at follow-up. Results were statistically significant. In conclusion, this study reveals the positive effects of caregiver training in the post-acute care management of stroke survivors at home.
\end{abstract}

\section{Introduction}

Stroke is the leading cause of adult disability which is inadequately addressed in some cases due to resource constraints. 1 Patient goes to the remote village after acute care management in the hospital with incomplete recovery and profound disability. Caregiver to manage the patient at home is usually not trained or briefed on physiotherapy or nursing care. Most of the patients return to next visit with many complications.

This study was aimed to find out the importance of caregiver briefing during discharge on outcome of stroke survivor.

\section{Materials and Methods}

In keeping with the pragmatic trial design, eligibility criteria were deliberately broad and inclusive. Patients were eligible for participation if they had a confirmed primary diagnosis of new stroke (ischemic or intracerebral hemorrhage; first or recurrent stroke); were medically stable, likely to return home with residual disability.

We have randomly selected 119 patients who were divided into two equal groups. Out of those, 60 patients were discharged within one week without any formal briefing or written instruction to the caregivers. Written and verbal instructions were given to the caregivers of 59 patients who stayed more than one week in the hospital. Informed consents were taken from all patients or their caregivers. Six patients in Group A and 9 patients in Group B were dropped during follow-up.

Caregivers of the first group were briefed on post-discharged management in Bangladesh's perspective during hospital stay by stroke unit team members that not necessarily correlate with London Structured Caregivers Training Course (LSCTC). Items demonstrated included diet, nutrition and feeding, continence care, bed positioning, activities of daily living (ADL), speech therapy, swallow therapy, physiotherapy. All the patients were asked to return after two months for follow-up.

Demographic profiles were recorded on age sex, occupation, type of stroke and major comorbidities like hypertension and diabetes mellitus. Simple disability parameter of Barthel indexes were recorded from all patients during discharge and at follow-up after two months.

\section{Results}

More than $75 \%$ of patients were above 60 years (Figure 1). Among the type of strokes, $71 \%$ were infarction. The rest $29 \%$ were of hemorrhagic type. Co-morbidities were hypertension $(52 \%)$, diabetes $(7 \%)$ and both (30\%) (Table I).

Barthel index in Group A during discharge were $6.2 \pm 4.0$ and at follow-up were $56.3 \pm 9.0$ (Table II). The index in Group B were $4.9 \pm 4.0$ during discharge and $28.2 \pm 7.8$ at follow-up. 


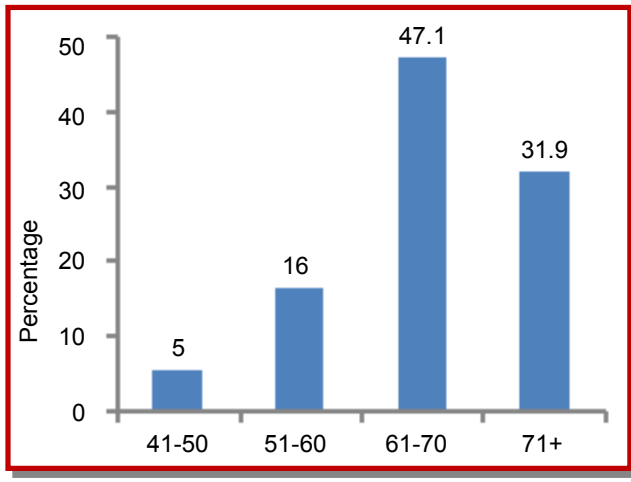

Figure 1: Distribution of age of patients

\section{Discussion}

National Stroke Association survey in USA found that stroke survivors often do not reach their rehabilitation goals, and lack of information is a major barrier to continued recovery: $38 \%$ of 523 long-term stroke survivors reported a lack of information about community and rehabilitation resources. 1

Inadequate provision of information is predictive of poor quality of life in stroke patients and their families. 2

Forster and colleagues (2001) reviewed 9 studies of educational intervention. 3 The authors excluded trials in which information giving was only one component of a more complex rehabilitation intervention (e.g., family support worker trials). There is some evidence that combining information with educational sessions improves knowledge and is more effective than providing information alone. 3

As the patient progresses from hospital-based rehabilitation to the community, involvement of caregivers in rehabilitation becomes increasingly important. Formal training of caregivers in delivery of care reduces personal costs and improves quality of life. $\underline{4}$

Evans et _al, ${ }^{5}$ mentioned that rehabilitation services are effective in improving short-term survival, func-tional ability and the most independent discharge location. One systematic review looked at caregiver support intervention and found that social support improved patient outcomes and family functioning. ${ }^{6} \mathrm{~A}$ comparison of passive versus active information intervention determined that there was no significant effect on the number of cases of anxiety or depression in patients, carer mood or satisfaction or death. A qualitative analysis found no strong evidence of an effect on other outcomes. Meta-analyses showed a significant effect from information therapy on patient and carer knowledge, one aspect of patient satisfaction, and patient depression scores.
An evidence-based educational program for stroke survivors after discharge home described 39 comprehensive educational guidelines. 7 The program recommended that educational programs provided to stroke survivors and their families be interactive, interdisciplinary, and focused on identified needs.

Training caregivers during patients' rehabilitation reduced costs and caregiver burden while improving psychosocial outcomes in caregivers and patients at one year. $\underline{4}$

The TRACS trial aimed to enhance patients' functional recovery, and reduce caregivers' burden by providing an in-patient, structured caregiver training program, the LSCTC. The TRACS trial did not demonstrate any benefit of this intervention for patients or caregivers.

There was no difference between the LSCTC and usual with respect to improving functional independence of patients who have had a stroke, reducing caregivers' burden, or improving other physical and psychological outcomes, nor is it cost effective when compared with usual care. 8

Families and caregivers should be educated in the care of these patients. The family and caregiver education may include; preventing recurrent stroke, signs and symptoms of potential complications and psychological dysfunction, medication administration, assisted ADL tasks (e.g., transfers, bathing, positioning, dressing, feeding, toileting, and grooming), swallowing techniques, nutrition and hydration, care of an indwelling bladder catheter, skin care, contractures, use of a feeding tube, home exercises (range of motions) and sexual functioning. 9

Caregivers were often invited to observe therapy or care being provided by professionals but had few opportunities to make sense of, or to develop knowledge and stroke-specific skills provided by the LSCTC. Where provided, caregiver training came very late in the inpatient stay. Assessment and development of caregiver competence was not commonly observed. Structured caregiver training programs such as the LSCTC are unlikely to be practical in settings with short inpatient stays. 
Stroke units where early supported discharge is in place potentially offer a more effective vehicle for introducing competency based caregiver training. $\underline{10}$

If the current discharge planning relies on the availability of a caregiver at home after discharge from inpatient stroke rehabilitation then it may be worthwhile these caregivers in the inpatient rehabilitation process to prepare them. More resources should be available to support caregivers in the community. $\underline{11}$

In Bangladesh, we have very little scope to provide the stroke survivors with trained caregivers like LSCTC due to resource constraints. In this study we have provided the caregivers with short briefing and written instructions that does not necessarily correlate with LSCTC about nursing care and physiotherapy at home and the compared outcome with those who have very early discharge and did not receive any caregivers instructions. In this study, we have used simple parameter of Barthel index for outcome assessment. The Barthel index measures the patient's degree of independence in performing ADL, and is the most frequently used measure of ADL competence in clinical stroke trials. $\underline{\mathbf{1 2 - 1 4}}$

\section{Conclusion}

Structured training of caregiver provided during discharge of hospital admitted stroke patient has positive effect on the outcome of survivor.

\section{References}

1. Jones F. Strategies to enhance chronic disease selfmanagement: How can we apply this to stroke? Disabil Rehabil. 2006 Jul 15-30; 28: 841-47.

2. O'Mahoney PG, Rodgers H, Thomson RG, Dobson RG, James OW. Satisfaction with information and advice received by stroke patients. Clin Rehabil. 1997; 11: 168-72.

3. Forster A, Smith J, Young J, Knapp P, House A, Wright J. Information provision for stroke patients and their caregivers. Cochrane Database Syst Rev.
2001: CD001919.

4. Kalra L, Evans A, Perez I, Melbourn A, Patel A, Knapp M, et al. Training carers of stroke patients: Randomized controlled trial. BMJ. 2004; 328: 1099.

5. Evans RL, Connis RT, Hendricks RD, Haselkorn JK. Multidisciplinary rehabilitation versus medical care: A meta-analysis. Soc Sci Med. 1995; 40: 1699 706.

6. Bhogal SK, Teasell RW, Foley NC, Speechley MR. Community reintegration after stroke. Top Stroke Rehabil. 2003; 10: 107-29.

7. Ostwald K, Davis S, Hersch G, Kellet C, Godwin K. Evidence-based educational guidelines for stroke survivors after discharge home. J Neurosci Nurs. 2008; 40: 173-79, 91.

8. Dickerson AJ, Young J, Patel A, Kalra L, Nixon J, et al. A structured training program for caregivers of inpatients after stroke (TRACS): A cluster randomized controlled forster trial and cost-effectiveness analysis. Lancet 2013; 382: 2069-76.

9. American Heart Association/American Stroke Association (VA/DOD) Clinical Practice Guidelines for the management of stroke rehabilitation, Version 2.0, 2010.

10. Clarke DJ, Hawkins R, Sadler E, Harding G, McKevitt C, Godfrey M, et al. Introducing structured caregiver training in stroke care: Findings from the TRACS process evaluation study. BMJ Open. 2014; 4: e004473.

11. Tanwir S, Montogomery S, Chari V, Nesathurai S. Stroke rehabilitation, availability of a family member as caregiver and discharge destination. Eur J Phys Rehabil Med. 2014; 50: 355-62.

12. Mahoney FT, Barthel DW. Functional evaluation: Barthel index. Md State Med J. 1965; 14: 61-65.

13. Sulter G, Steen C, De Keyser J. Use of the Barthel index and modified Rankin scale in acute stroke trials. Stroke 1999; 30: 1538-41.

14. Lyden P, Broderick J, Mascha L. Reliability of the Barthel index outcome measure selected for the NINDS t-PA stroke trial. In: Thrombolytic therapy in acute ischemic stroke III. Yamaguchi T, Mori E, Minematsu K, del Zoppo G, eds. Tokyo, SpringerVerlag, 1995, pp 327-33. 